Abanico Veterinario. Enero-Diciembre 2020; 10:1-13. http://dx.doi.org/10.21929/abavet2020.26

Artículo Original. Recibido: 03/07/2020. Aceptado: 19/10/2020. Publicado: 20/11/2020. Clave: 2020-53.

\title{
Caracterización química de extracto alcohólico de hoja de guayaba (Psidium guajava) y su efecto como inhibidor de movilidad para Escherichia coli 0157:H7
}

\author{
Chemical characterization of alcoholic extract of guava leaf ( Psidium guajava) and its \\ effect as a mobility inhibitor for Escherichia coli O157:H7
}

\section{Mónica Silva-Vega ${ }^{1^{*} I D}$, Rómulo Bañuelos-Valenzuela ${ }^{1 * * ~ I D, ~ L u c i ́ a ~ D e l g a d i l l o-R u i z ~}{ }^{2}$ ID, Perla Gallegos-Flores ${ }^{2}$ ID, Carlos Meza-López ${ }^{1 \text { ID }}$, Benjamín Valladares-Carranza ${ }^{3 \text { ID, }}$ Francisco Echavarría-Cháirez ${ }^{4} \mathrm{ID}$}

\begin{abstract}
${ }^{1}$ Unidad Académica de Medicina Veterinaria y Zootecnia, Universidad Autónoma de Zacatecas. ${ }^{2}$ Unidad Académica de Ciencias Biológicas, Universidad Autónoma de Zacatecas. Avenida preparatoria s/n colonia Hidráulica, CP. 98068, Zacatecas, Zacatecas, México. ${ }^{3}$ Facultad de Medicina Veterinaria y Zootecnia. Centro de Investigación y Estudios Avanzados en Salud Animal. Universidad Autónoma del Estado de México. Toluca, México. ${ }^{4}$ Instituto nacional de investigaciones forestales, agrícolas y pecuarias. Campo experimental Zacatecas, México. *Autor responsable: Mónica Silva-Vega. ${ }^{* *}$ Autor de correspondencia: Rómulo Bañuelos-Valenzuela. Carretera Panamericana Fresnillo-Zacatecas s/n, Centro, CP. 98500 Víctor Rosales, Zacatecas, México. msilva58@hotmail.com, apozolero@hotmail.com, delgadillolucia@gmail.com, perla_gf17@hotmail.com, carmezlop@yahoo.com.mx,
\end{abstract} benvac2004@yahoo.com.mx, fechava1@yahoo.com

\section{RESUMEN}

El objetivo fue caracterizar y determinar el efecto inhibitorio de movilidad en Escherichia coli O157:H7 de extractos de hojas de guayaba (Psidium guajava). Se han buscado nuevas alternativas de origen natural "extractos de plantas" para eliminar la colonización de bacterias patógenas en animales y prevenir la contaminación de carne. El extracto de hoja de guayaba (Psidium guajava) tiene actividad antibacteriana de amplio espectro, debido al principio activo quercetina. E. coli O157:H7 enterohemorragica, es un patógeno de importancia en salud pública, que puede causar síndrome urémico hemolítico, además los rumiantes son reconocidos como el principal hospedero de E. coli O157: $\mathrm{H} 7$. El extracto fue preparado con hojas de guayaba en etanol al 70\%, obteniendo un extracto crudo (Extracto A) y uno concentrado mediante el uso del equipo soxhlet (Extracto B). Se determinó la composición química por cromatografía de gases. Se muestrearon rumiantes lactantes con síndrome diarreico, las muestras fueron transportadas en medio Stuart. Las bacterias se aislaron en medio Mac Conkey y posteriormente fueron sembradas en medio CHROMagar $^{\text {TM }} 0157$ para la identificación de E. coli O157:H7. Se realizaron pruebas de movilidad de $E$. coli 0157:H7 en medio SIM, con extracto de hoja de guayaba y como referencia se utilizaron concentraciones de carvacrol de $0.3,1$ y $5 \mathrm{mM}$ y quercetina 205, 102 y $51 \mathrm{mM}$. Se identificaron $78 \mathrm{E}$. coli O157: $\mathrm{H} 7$, las cuales mostraron inhibición en la movilidad a diferentes concentraciones de carvacrol, en quercetina $205 \mathrm{mM}$ y $102.5 \mathrm{mM}$ y en los extractos A y B. Se concluye que el extracto alcohólico de hojas de guayaba y su compuesto en mayor proporción (quercetina) son efectivos en la inhibición de movilidad de E. coliO157 H7.

Palabras clave: Extractos, carvacrol, quercetina, inhibición. 


\section{ABSTRACT}

The objective was to characterize and determine the mobility inhibitory effect in Escherichia coli O157: $\mathrm{H} 7$ of extracts of guava leaves (Psidium guajava). New alternatives of natural origin "plant extracts" have been sought to eliminate colonization of pathogenic bacteria in animals and prevent contamination of meat. Guava leaf extract (Psidium guajava) has broad-spectrum antibacterial activity, due to the active ingredient quercetin. E. coli O157: $\mathrm{H} 7$ enterohemorrhagic, is a pathogen of great importance in public health, which can cause hemolytic uremic syndrome, and ruminants are recognized as the main host of E. coli O157: $\mathrm{H} 7$. The extract was prepared with guava leaves in $70 \%$ ethanol, obtaining a crude extract (Extract $A$ ) and a concentrated extract using the soxhlet equipment (Extract B). Its chemical composition was determined by gas chromatography. Nursing ruminants with diarrheal syndrome were sampled, the samples were transported in Stuart medium. The bacteria were isolated in Mac Conkey medium and subsequently seeded in CHROMagar ${ }^{\mathrm{TM}} 0157$ medium for the identification of E. coli O157:H7. Mobility tests of E. coli O157: $\mathrm{H} 7$ were carried out in SIM medium, with guava leaf extract and as a reference, concentrations of carvacrol of 0.3, 1 and $5 \mathrm{mM}$ and quercetin 205, 102 and $51 \mathrm{mM}$ were used. 78 E. coli O157: $\mathrm{H} 7$ were identified, which showed inhibition in mobility at different concentrations of carvacrol, in quercetin $205 \mathrm{mM}$ and $102.5 \mathrm{mM}$ and in extracts A and B. It is concluded that the alcoholic extract of guava leaves and its compound in a greater proportion (quercetin) they are effective in inhibiting the mobility of E. coli $\mathrm{O} 157 \mathrm{H} 7$.

Keywords: Extracts, carvacrol, quercetin, inhibition.

\section{INTRODUCCIÓN}

Escherichia coli O157, es un patógeno importante en salud pública, que puede llegar a producir toxina Shiga (STEC) (Kaper et al., 2004). Los productos STEC son transmitidos a través de los alimentos, especialmente el serotipo O157:H7. Las enfermedades causadas al humano por el serotipo que produce STEC, van desde diarrea leve a colitis hemorrágica y síndrome urémico hemolítico (SHU), que por lo general afecta a niños, pacientes de edad avanzada e inmunocomprometidos (Rodríguez-Angeles, 2002). La patogenicidad de STEC reside en diferentes factores de virulencia, incluyendo las toxinas Shiga (Stx1 y Stx2), intimina, enterohemolisina y el autoaglutinantes STEC adhesina (AEA) (Gyles, 2007).

Se ha reportado que los rumiantes domésticos como vacas, ovejas y cabras son portadores asintomáticos, que pueden portar STEC y E. coli O157:H7 en sus heces, por lo que se consideran reservorios naturales de estos patógenos (Blanco et al., 2004; Milton et al., 2018; Iweriebor et al., 2015; Bolukaoto et al., 2019). Para eliminar la colonización de bacterias patógenas en animales y prevenir la contaminación de la carne, existe una variedad de agentes químicos antimicrobianos que están disponibles para la terapéutica en ganado; sin embargo, investigadores en nutrición animal han reportado que con el aumento del uso de agentes antimicrobianos en animales y seres humanos, ha aumentado la prevalencia de cepas resistentes (Cattoir y Leclercq, 2017; Kim et al., 2019). Los genes CTX-M $\beta$-lactamasas han sido reportados en E. coli, a partir de ganado con fines alimenticios en todo el mundo; levantando una amenaza potencial para la salud pública (Wittum et al., 2010; Botelho et al., 2015; Vitas et al., 2018).

A partir del aumento de cepas resistentes a antibióticos, se han buscado nuevas alternativas de origen natural, como los extractos de plantas; por ejemplo, el extracto de 
hoja de guayaba (Psidium guajava), que tiene actividad antibacteriana de amplio espectro (Martínez et al., 1997; Bermúdez-Vásquez et al., 2019). Rattanachaikunsopon y Phumkhachorn (2010), reportan que el extracto acuoso de hoja de guayaba mostró actividad antibacteriana en un $35 \%$ de los casos, el alcohólico en un $65 \%$ y el cetónico en el 100\%, contra bacterias patógenas; incluyendo Bacillus stearothermophilus, Brochothrix thermosphacta, E. coli O157:H7, Listeria monocytogenes, Pseudomonas fluorescens, Salmonella enterica, Staphylococcus aureus y Vibrio cholerae.

El extracto acuoso de las hojas de guayaba disminuye la producción de toxinas lábiles de E. coli y del cólera (Birdi et al., 2010). Echemendía y Morón (2004) en su ensayo clínico concluyeron que la tintura al $20 \%$ de hoja de Psidium guajava tiene efecto antidiarreico y Lozoya et al. (2002) evaluaron el polvo de las hojas secas, comprobándose este efecto. Se han aislado diversos compuestos químicos, a partir del extracto de hoja de guayaba, como son: el triterpenoide pentacíclico, el ácido guajanoico; así como, B-sitosterol, uvaol, ácido oleanólico y ácido ursólico y quercetina (Biswas et al., 2013), para comprobar el efecto antibacteriano que presentan las hojas de guayaba. Por lo que el objetivo del presente trabajo fue la caracterización y determinación del efecto inhibitorio de movilidad en E. coli O157:H7, de extractos de hojas de guayaba (Psidium guajava).

\section{MATERIAL Y MÉTODOS}

\section{Obtención del extracto alcohólico de hojas Psidium guajava}

Para cada extracto se utilizó etanol al 70\% J.T. Baker, en una relación de 25 gramos de la muestra molida por cada $200 \mathrm{~mL}$ de solvente. La mezcla fue colocada y sellada en frascos color ámbar de un litro, se homogenizo vigorosamente por $10 \mathrm{~min}$; el extracto se dejó reposar durante un mes a temperatura ambiente. El sobrenadante fue pasado a través de papel filtro (Whatman No. 2), para remover los restos del polvo de la planta (Pesewu et al., 2008). Una parte del extracto preparado se empleó para concentrarlo mediante el uso del equipo soxhlet durante una hora, recuperando la mitad del volumen inicial, llamándolo Extracto B. El extracto A se le nombró al extracto más diluido, o como se obtuvo después de la filtración.

\section{Composición química de los extractos de hoja de guayaba por cromatografía de gases}

La composición química se determinó mediante un cromatógrafo de gases (CG; Agilent Technologies serie $6890 \mathrm{~N}$ fabricado en U.S.A), con una columna polar DB_WAXetr, a $250{ }^{\circ} \mathrm{C}$ y 12.13 psi con un flujo de He $36.5 \mathrm{~mL} \mathrm{~min}^{-1}$ después de la inyección. Las condiciones para la columna fueron: temperatura inicial $50{ }^{\circ} \mathrm{C}$, de cero a dos min, aumentando de 10 en $10^{\circ} \mathrm{C}$ hasta llegar a $250^{\circ} \mathrm{C}$, manteniendo la temperatura constante por $5 \mathrm{~min}$, para luego descender a $50^{\circ} \mathrm{C}$ por dos min con un flujo de He de $1.6 \mathrm{~mL} \mathrm{~min}^{-1}$ a una presión de 12.13 psi y una velocidad promedio de $25 \mathrm{~cm} \mathrm{~s}^{-1}$, utilizando un detector de flama ionizante (FID) a una temperatura de $210{ }^{\circ} \mathrm{C}$, con un flujo de $\mathrm{H}_{2}$ de $40 \mathrm{~mL} \mathrm{~min}^{-1}$ 
y un flujo de aire de $450 \mathrm{~mL} \mathrm{~min}^{-1}$. Para el corrimiento de muestras en el cromatógrafo se emplearon los estándares de carvacrol y timol (Sigma-Aldrich) (Bañuelos-Valenzuela et al., 2018).

\section{Determinación de la dosis mínima hemolítica de los extractos}

Se extrajeron $10 \mathrm{~mL}$ de sangre en un tubo heparinizado; se centrifugó el tubo con la sangre sin coagular a $2500 \mathrm{rpm} \times 10 \mathrm{~min}$ a $10^{\circ} \mathrm{C}$, removieron la fracción del suero con una pipeta; posteriormente se realizaron tres lavados con un regulador de lavado [PBS al 50\% (v/v) y glucosa al 2.25\% (w/v)] (López et al., 2017); luego se centrifugó en cada lavado a $2500 \mathrm{rpm} \times 10 \mathrm{~min}$ a $10^{\circ} \mathrm{C}$. El paquete de eritrocitos se recuperó y se resuspendió hasta una concentración del $0.1 \%(\mathrm{v} / \mathrm{v})$ con un regulador de suspensión, que consiste en PBS al 50\% (v/v) glucosa al $2.25 \%(\mathrm{w} / \mathrm{v})$ y gelatina al $0.05 \%(\mathrm{w} / \mathrm{v})$. Se realizó por triplicado el ensayo en placas de 96 pozos de fondo $\mathrm{U}$; se mezclaron $100 \mu \mathrm{L}$ de la suspensión de eritrocitos al $1 \%$ con $100 \mu \mathrm{L}$ de la suspensión de cada extracto y con diluciones de 1:10, 1:100 y 1:1000.

El control negativo únicamente se inoculó $100 \mu \mathrm{L}$; este mismo procedimiento se realizó para cada principio activo en sus diferentes concentraciones (carvacrol, timol y quercetina), de la suspensión de eritrocitos al 1\%. Para el control positivo se mezclaron $100 \mu \mathrm{L}$ de la suspensión de eritrocitos al $1 \%$ con $100 \mu \mathrm{L}$ de Tritón $\mathrm{X}$ al $1 \%$; se observaron los cambios en la suspensión de eritrocitos cada hora, hasta cumplir $24 \mathrm{~h}$. Con base a los controles positivo y negativo se determinó si había actividad hemolítica del extracto sobre los eritrocitos.

Para el corrimiento de muestras en el cromatógrafo, se emplearon los estándares de carvacrol y timol, de la marca SIGMA grado reactivo. Se concentró el extracto de hoja de guayaba (40 mg/mL) mediante ebullición (Ext A).

\section{Identificación de bacterias en medio cromogénico CHROMagar ${ }^{\mathrm{TM}}$}

Se obtuvieron hisopados rectales provenientes de rumiantes lactantes, con presencia de síndrome diarreico, menores de 21 días de edad y con la seguridad de haber ingerido calostro. La colecta de las muestras se realizó vía rectal con un hisopo estéril, se etiquetaron y transportaron en medio Stuar ${ }^{\circledR}$ elaborado en México D.F; cada muestra se sembró en la caja petri con agar MacConkey. Se tomó una colonia para proseguir con la identificación, la cual se realizó mediante una siembra por estría en placa en medio cromogénico CHROMagar ${ }^{\mathrm{TM}}$ O:157, para E. coli O:157:H7 (Moyne et al., 2011). Luego seleccionaron 78 cepas bacterianas de heces de rumiantes lactantes con síndrome diarreico menores de tres meses, identificadas como E. coli 0157:H7 en CHROMagar ${ }^{\mathrm{TM}}$ las cuales presentaron coloración rosa malva, debido a sustratos cromogénicos en el medio; permitiendo así la identificación presuntiva de la placa de aislamiento primario y la diferenciación de otros organismos (Hirvonen et al., 2012; Lara et al., 2019). 


\section{Preparación del medio SIM en tubo con extracto}

Se preparó medio SIM para cada tipo de estándar y extracto. Para la preparación del medio SIM, se pesaron $30 \mathrm{~g}$ de agar por cada litro de agua destilada; el agar fue esterilizado en autoclave a $121^{\circ} \mathrm{C}$ por $15 \mathrm{~min}$. Se dejó enfriar el agar a una temperatura de $35{ }^{\circ} \mathrm{C}$ aproximadamente, para adicionar el extracto y el estándar correspondiente; posteriormente se adicionaron $4 \mathrm{~mL}$ de la mezcla anterior en tubos estériles de $10 \mathrm{~mL}$.

Para cada bacteria se realizó una serie de tubos por triplicado, como se describe a continuación: Control (sin extracto), Carvacrol (C $0.3 \mathrm{mM}, \mathrm{C} 1 \mathrm{mM}$ y C $5 \mathrm{mM}$ ), Ext A (extracto guayaba diluido), Ext B (extracto guayaba concentrado), Q 205 (quercetina 205 $\mathrm{mM}$ ), Q 102 (quercetina $102 \mathrm{mM}$ ), Q 51 (quercetina $51.25 \mathrm{mM}$ ) y $\mathrm{OH}$ (control alcohol).

\section{Pruebas de movilidad bacteriana}

Cada una de las bacterias identificadas por CHROMagar ${ }^{\mathrm{TM}} \mathrm{O}: 157$, fue sembrada en agar base por estría, con el objetivo de obtener una sola colonia asilada para posteriormente realizar la siembra en tubo. La siembra en tubo se realizó mediante picadura, que consiste en tomar una colonia aislada de bacterias y hacer una picadura en el medio SIM, atravesando el agar hasta el fondo del tubo; al medio se preparó con los extractos, estándares y control. Una vez terminada la siembra por picadura, todas las muestras permanecieron a una temperatura de $37^{\circ} \mathrm{C}$, en una incubadora Thermo $\AA$ durante un periodo de $24 \mathrm{~h}$.

Las condiciones de siembra se hicieron con debida esterilidad para evitar contaminación; estas se realizaron en una campana de flujo laminar (Lab tech $\AA$ ). La movilidad bacteriana fue medida, usando un método cualitativo; a) motilidad positiva (+): presencia de turbidez difusa $o$ total en el medio. b) motilidad negativa (-): ausencia o presencia leve de crecimiento, solo en el sitio de la picadura.

\section{Análisis estadístico}

El análisis estadístico realizado fue el de tablas de contingencia de dimensión $2 \times 2$, entre las variables extracto $A$ y extracto $B$ vs carvacrol $0.3 \mathrm{mM}$, carvacrol $1 \mathrm{mM}$, carvacrol 5 $\mathrm{mM}$, quercetina $205 \mathrm{mM}$, quercetina $102 \mathrm{mM}$ y quercetina $51 \mathrm{mM}$. Los criterios utilizados fueron las pruebas de independencia de $\chi^{2}$ (prueba ji cuadrada), considerando un nivel de significancia de $p<0.05$ y un intervalo de confianza del $95 \%$ (Good, 2000). Los datos fueron capturados en Excel y analizados en el programa Statistical Package for the Social Sciences (SPSS por sus siglas en inglés) versión 17.

\section{RESULTADOS Y DISCUSIÓN}

En la tabla 1, se muestra la presencia de los principios activos carvacrol y timol en los extractos alcohólicos de hojas de guayaba, así como su concentración por cromatografía de gases. 
Tabla 1. Resultados de la cromatografía de gases expresado en unidades de concentración $\mathrm{mg} / \mathrm{mL}$

\begin{tabular}{ccc}
\hline $\begin{array}{c}\text { Extracto de hoja de } \\
\text { Guayaba }\end{array}$ & Carvacrol & Timol \\
\hline Extracto A & 3.0869 & 1.5130 \\
Extracto B & 0 & 0.3525 \\
\hline
\end{tabular}

Ambos principios activos (carvacrol y timol), estuvieron presentes en el extracto A; mientras que el extracto $B$, sólo se identificó el timol. El principio activo de mayor concentración en los extractos fue el carvacrol con $3.0869 \mathrm{mg} / \mathrm{mL}$ en el extracto A. Las plantas medicinales son comúnmente ricas en terpenos (carvacrol, citral, linalol y geraniol) y compuestos fenólicos, compuestos eficaces como aditivos alimentarios (Nile et al., 2017).

El modo primario de acción antibacteriana del timol no se conoce completamente, pero se cree que implica la disrupción de la membrana externa e interna y la interacción con proteínas de membrana y dianas intracelulares. Los estudios de Wang y Yam (2018) han demostrado que el timol interactúa con las membranas celulares, y estas interacciones afectan la permeabilidad de la membrana bacteriana. Para el efecto hemolítico y con base a los controles positivo y negativo, se determinó si había actividad hemolítica de los extractos de hojas de guayaba y los principios activos sobre los eritrocitos. En la tabla 2 se observó que para carvacrol a una concentración de $0.3 \mathrm{mM}$, no se presentó hemolisis, en los extractos $\mathrm{A}$ y $\mathrm{B}$ sólo hay hemolisis hasta la dilución 1:10. Por último, para quercetina la concentración mínima de hemolisis fue en dilución 1:10 de sus tres concentraciones. Como menciona López et al. (2017) el término hemólisis hace referencia al proceso de destrucción de los eritrocitos, que genera la liberación de los componentes intraeritrocitarios; por lo tanto, la prueba de hemólisis se utiliza para conocer el efecto provocado sobre la célula eritrocitaria al enfrentarla con los extractos a diferentes concentraciones, que es lo que se pretendió con este experimento. La inhibición de hemólisis se debe a los componentes bioactivos, tipo flavonoides y compuestos fenólicos presentes en los extractos de guayaba.

Los eritrocitos pueden cambiar su forma normal a la forma de equinocitos o estomatocitos, lo cual depende de factores citoplasmáticos, entre ellos el pH (Gedde et al., 1997). Las altas concentraciones de flavonoides hallados en frutas como el mango (García Bacallao et al., 2001), hojas de guayaba (Rodríguez et al., 2013) y flavonoides obtenidos de maqui (Aristotelia chilensis) con propiedades antioxidantes, son inductores de equinocitos (Gironés-Vilaplana et al., 2012; Durán et al., 2013). 
Tabla 2. Actividad hemolítica de los extractos de hoja de guayaba

\begin{tabular}{ccccc}
\hline Dilución & $\begin{array}{c}\text { Solución } \\
\text { muestra }\end{array}$ & $\mathbf{1 : 1 0}$ & $\mathbf{1 : 1 0 0}$ & $\mathbf{1 : 1 0 0 0}$ \\
\hline C $0.3 \mathrm{mM}$ & + & - & - & - \\
C $1 \mathrm{mM}$ & + & + & - & - \\
C 5 mM & + & + & + & - \\
EXT A & + & + & - & - \\
EXT B & + & + & - & - \\
Q $205 \mathrm{mM}$ & + & + & - & - \\
Q $102 \mathrm{mM}$ & + & + & - & - \\
Q 51 mM & + & + & - & - \\
\hline
\end{tabular}

(+) presenta hemolisis; (-) ausencia de hemolisis

Con el uso del CHROMagar se identificó E. coli O:157 H7, diferenciándose por el color de la colonia en el medio, presenta una coloración rosa malva (Lara et al., 2019). Este medio inicialmente se utilizaba en la industria alimentaria para la liberación rápida de alimentos libres de patógenos, pero actualmente se encuentra aprobado para el análisis de muestras clínicas y ha sido empleado en diversos estudios (Bettelheim, 1998; Tang et al., 2014; Gutierrez et al., 2016; Parsons et al., 2016). Se identificaron 78 cepas de E. coli O157:H7 mediante CHROMagar ${ }^{\mathrm{TM}}$ selectivo, y estas bacterias fueron inoculadas en medio SIM.

Los resultados de la movilidad in vitro de los extractos de hoja de guayaba y los estándares (principios activos de hoja de guayaba) sobre las bacterias, se observan en la tabla 3. Este efecto se evaluó cualitativamente por la presencia o ausencia de turbidez en el tubo; los resultados mostraron que los estándares de carvacrol tienen amplia actividad antibacteriana, frente a 65 microorganismos; inhibiendo en 56 bacterias el crecimiento bacteriano a una concentración de $5 \mathrm{mM}$ (tabla 3); en relación con el extracto A de guayaba que presentó efecto en la inhibición de la movilidad de 62 bacterias; mientras que el extracto B inhibe 46 bacterias. Quercetina a una concentración de 51 $\mathrm{mM}$, presentó la mayor inhibición de movilidad en 60 bacterias.

Tabla 3. Resultados totales de movilidad bacteriana

\begin{tabular}{cccccccccc}
\hline & C 5 $\mathbf{~ m M}$ & C 1 $\mathbf{~ m M}$ & C 0.3 $\mathbf{~ m M}$ & EXT A & EXT B & Q 205 & Q 102 & Q 51 & OH \\
\hline+ & 56 & 60 & 65 & 62 & 46 & 59 & 51 & 60 & 0 \\
- & 22 & 18 & 13 & 16 & 32 & 19 & 27 & 18 & 78 \\
\hline
\end{tabular}

(+): Presencia de turbidez difusa o total en el medio. (-): Ausencia o presencia leve de crecimiento.

Gallegos- Flores et al. (2019) reportaron que el carvacrol a una concentración de $0.3 \mathrm{mM}$ disminuye la movilidad de la cepa determinada con la técnica de Wertern Blot, donde observaron la disminución de la síntesis de flagelina; esta proteína se encuentra en un $8 \%$ de la proteína total celular. Estos resultados difieren de los reportados en la tabla 2, 
debido principalmente a que la concentración de $0.3 \mathrm{mM}$, no inhibió la movilidad en el $100 \%$ de las bacterias E. coli O157:H7.

Desde el punto de vista de Gallegos- Flores et al. (2019), las células de E. coli crecen en presencia de carvacrol a una concentración de $5 \mathrm{mM}$ sin síntesis de flagelos, provocando que el microorganismo crezca sin movilidad; es decir, cuando la célula bacteriana está sujeta a un estrés ocasionado por sustancias tóxicas y se encuentra en riesgo su supervivencia; la cual es capaz de suprimir la producción de la proteína flagelina y conservar energía para otras funciones celulares, que pueden por lo tanto, ser una táctica de supervivencia; sin embargo, a una concentración mayor de $5 \mathrm{mM}$ la bacteria cesa inmediatamente la movilidad y ocurre muerte celular; observándose que la concentración de $5 \mathrm{mM}$ es la que presenta mayor número de bacterias que inhibieron el crecimiento.

La interacción carvacrol-quercetina que se encuentra presente en el extracto A de hojas de guayaba, presenta una mayor inhibición en la movilidad de las bacterias, siempre y cuando las concentraciones de carvacrol y quercetina sean las antes señaladas (tabla 4). Ambos casos son significativos, pero se distinguen una magnitud de comparación mayor del extracto A con carvacrol y quercetina, debido a los compuestos químicos presentes. Menor magnitud de Chi cuadrada representa mayor semejanza al extracto; por lo tanto, el extracto B representa más semejante al estándar de quercetina, pero no tiene el efecto de inhibición.

Tabla 4. Eficiencia de los extractos

\begin{tabular}{|c|c|c|c|c|c|c|c|c|c|c|}
\hline \multirow{2}{*}{\multicolumn{2}{|c|}{ Concentración }} & & \multicolumn{4}{|c|}{ Extracto $\mathrm{A}$} & \multicolumn{4}{|c|}{ Extracto B } \\
\hline & & & & $\chi^{2}$ & $\mathbf{z}$ & $p$ & & $\chi^{2}$ & $\mathbf{z}$ & $p$ \\
\hline \multirow{6}{*}{$\begin{array}{l}\text { Carvacrol } \\
(\mathrm{mM})\end{array}$} & \multirow{2}{*}{0.3} & + & 127 & 81.41 & \multirow{2}{*}{7.84} & \multirow{2}{*}{0.0001} & 111 & 71.15 & \multirow{2}{*}{5.28} & \multirow{2}{*}{0.0001} \\
\hline & & - & 29 & 18.59 & & & 45 & 28.85 & & \\
\hline & \multirow{2}{*}{1} & + & 122 & 78.21 & \multirow{2}{*}{7.04} & \multirow{2}{*}{0.0001} & 106 & 67.95 & \multirow{2}{*}{4.48} & \multirow{2}{*}{0.0001} \\
\hline & & - & 34 & 21.79 & & & 50 & 32.05 & & \\
\hline & \multirow{2}{*}{5} & + & 118 & 75.64 & \multirow{2}{*}{6.4} & \multirow{2}{*}{0.0001} & 102 & 65.38 & \multirow{2}{*}{3.84} & \multirow{2}{*}{0.0001} \\
\hline & & - & 38 & 24.36 & & & 54 & 34.62 & & \\
\hline \multirow{6}{*}{$\begin{array}{l}\text { Quercetina } \\
\quad(\mathrm{mM})\end{array}$} & \multirow{2}{*}{205} & + & 121 & 77.56 & \multirow{2}{*}{6.88} & \multirow{2}{*}{0.0001} & 105 & 67.31 & \multirow{2}{*}{4.32} & \multirow{2}{*}{0.0001} \\
\hline & & - & 35 & 22.44 & & & 51 & 32.69 & & \\
\hline & \multirow{2}{*}{102.5} & + & 113 & 72.44 & \multirow{2}{*}{5.6} & \multirow{2}{*}{0.0001} & 97 & 62.18 & \multirow{2}{*}{3.04} & \multirow{2}{*}{0.0023} \\
\hline & & - & 43 & 27.56 & & & 59 & 37.82 & & \\
\hline & \multirow{2}{*}{51.25} & + & 122 & 78.21 & \multirow{2}{*}{7.04} & \multirow{2}{*}{0.0001} & 106 & 67.95 & \multirow{2}{*}{4.48} & \multirow{2}{*}{0.0001} \\
\hline & & - & 34 & 21.79 & & & 50 & 32.05 & & \\
\hline
\end{tabular}

El carvacrol daña la membrana exterior de las bacterias gram negativas e incrementan la permeabilidad de la membrana citoplasmática que causa pérdidas de ATP, fuga de iones y lisis celular (Meira et al., 2017). El hecho de que las bacterias gram negativas flageladas en presencia de carvacrol no desarrollen flagelos, podría tener implicaciones para el uso de este compuesto como aditivo antibacteriano para productos alimenticios y/o para la generación de nuevos antibióticos; ya que si la célula bacteriana no presenta flagelos, 
esto disminuye o inhibe su mecanismo de patogenicidad, al ser menos capaz de adherirse a las células epiteliales del huésped.

Finalmente, el efecto de quercetina sobre la E. coli O157:H7 a una concentración de 102 $\mathrm{mM}$, resulta ser la óptima en la movilidad de las bacterias, teniendo el mismo efecto en la interacción quercetina-extracto B; esto puede ser atribuido a que el principal compuesto activo en las hojas de guayaba es el flavonoide quercetina, al cual se le atribuye un efecto antibacteriano (Echemendía y Morón, 2004).

\section{CONCLUSIÓN}

Se concluye que el extracto A de hoja de guayaba y su compuesto en mayor proporción (quercetina) son efectivos en la inhibición de la movilidad de E. coli O157:H7; por lo tanto lo hace una alternativa de origen natural para el tratamiento del síndrome diarreico en rumiantes.

\section{LITERATURA CITADA}

BAÑUELOS-VALENZUELA R, Delgadillo-Ruiz L, Echavarría-Cháirez F, Delgadillo-Ruiz O, Meza-López C. 2018. Composición química y FTIR de extractos etanólicos de Larrea tridentata, Origanum vulgare, Artemisa ludoviciana y Ruta graveolens. Agrociencia. 52(3): 309-321. http://www.scielo.org.mx/pdf/agro/v52n3/2521-9766-agro-52-03-309.pdf

BETTELHEIM KA. 1998. Reliability of CHROMagar® 0157 for the Detection of Enterohaemorrhagic Escherichia coli (EHEC) 0157 but Not EHEC Belonging to Other Serogroups. Journal of Applied Microbiology. 85:425-428. https://doi.org/10.1046/j.13652672.1998.853469.x

BERMÚDEZ-VÁSQUEZ MJ, Granados-Chinchilla F, Molina A. 2019. Chemical composition and antimicrobial activity of the essential oil of Psidium guajava and Cymbopogon citratus. Agronomía Mesoamericana. 30(1): 147-163. http://dx.doi.org/10.15517/am.v30i1.33758

BISWAS B, Rogers K, McLaughlin F, Daniels D, Yadav A. 2013. Antimicrobial activities of leaf extracts of guava (Psidium guajava L.) on two gram-negative and gram-positive bacteria. International journal of microbiology. 2013: 1-7. https://doi.org/10.1155/2013/746165

BIRDI T, Daswani P, Brijesh S, Tetali P and Natu A. 2010. Newer insights into the mechanismof action of Psidium guajava $\mathrm{L}$. leaves in infectious diarrhoea. $B M C$ complementary and alternative medicine. 33. https://link.springer.com/article/10.1186/1472-6882-10-33 
BLANCO M, Padola NL, Krüger A, Sanz ME, Blanco JE, González EA, Dahbi G, Mora A, Bernárdez MI, Etcheverría Al, Arroyo GH, Lucchesi PMA, Parma AE, Blanco J. 2004. Virulence genes and intimin types of Shiga-toxin-producing Escherichia coli isolated from cattle and beef products in Argentina. Int Microbiol. 7:269-76. http://revistes.iec.cat/index.php/IM/article/view/9482

BOLUKAOT JY, Kock MM, Strydom KA, Mbelle NM and Ehlers MM. 2019. Molecular characteristics and genotypic diversity of enterohaemorrhagic Escherichia coli O157: H7 isolates in Gauteng region, South Africa. Science of the Total Environment. 692: 297-304. https://doi.org/10.1016/j.scitotenv.2019.07.119

BOTELHO LAB, Kraychete GB, e Silva C, Lapa J, Regis DVV, Picão RC and Bonelli RR. 2015. Widespread distribution of CTX-M and plasmid-mediated AmpC $\beta$-lactamases in Escherichia coli from Brazilian chicken meat. Memórias do Instituto Oswaldo Cruz. 110(2): 249-254. https://doi.org/10.1590/0074-02760140389

CATTOIR V, Leclercq R. 2017. Resistance to Macrolides, Lincosamides, and Streptogramins. Antimicrobial Drug Resistance (pg 269-280). Springer, Cham. https://link.springer.com/chapter/10.1007/978-3-319-46718-4_18

DURÁN M, Montero P, Marrugo Y. 2013. Extractos metanólicos de corteza de guayaba (Psidium guajava L.) y mango (Mangifera indica L.): efecto citotóxico, antihemolítico y en la morfología de membrana de eritrocitos. Revista UDCA Actualidad \& Divulgación Científica. 16(2):327-334. https://repository.udca.edu.co/handle/11158/1744

ECHEMENDÍA SCE, Morón RFJ. 2004. Tintura de hojas de Psidium guajava L. enpacientes con diarrea aguda simple. Rev Cubana Plant Med. 9(3): 1-13. http://scielo.sld.cu/scielo.php?script=sci_arttext\&pid=S1028-47962004000300008

GALLEGOS-FLORES PI, Bañuelos-Valenzuela R, Delgadillo-Ruiz L, Meza-López C y Echavarría-Cháirez F. 2019. Actividad antibacteriana de cinco compuestos terpenoides: carvacrol, limoneno, linalool, a-terpineno y timol. Tropical and Subtropical Agroecosystems. 22:241-248.

https://pdfs.semanticscholar.org/c11b/a9ad9c764c58baaded8d9a5eded06c7c1513.pdf

GARCÍA BL, García GVL, Rojo DM, Sánchez GE. 2001. Plantas con propiedades antioxidantes. Revista cubana de investigaciones biomédicas. 20(3):231-235. http://scielo.sld.cu/scielo.php?script=sci_arttext\&pid=S0864-03002001000300011 
GEDDE MM, Davis DK, Huestis WH. 1997. Cytoplasmic pH and human erythrocyte shape. Biophysical journal. 72(3):1234-1246. https://www.cell.com/biophysj/pdf/S00063495(97)78770-8.pdf

GIRONÉS-VILAPLANA A, Mena P, García-Viguera C, Moreno DA. 2012. A novel beverage rich in antioxidant phenolics: Maqui berry (Aristotelia chilensis) and lemon juice. LWT - Food Science and Technology. 47(2): 279-286. https://doi.org/10.1016/j.Iwt.2012.01.020

GOOD P. 2000. Permutation tests. A practical guide to resampling methods for testing hypotheses. Second edition. Springer-Verlag, New York. Pp. 270. https://doi.org/10.1007/978-1-4757-3235-1

GUTIERREZ ME, Janes ME, Torrico DD, Carabante KM, Prinyawiwatkul W. 2016. Assessment of the ability of five culture media for the detection of Escherichia coli O157. International Journal of Food Science \& Technology, 51(8), 1910-1915. https://doi.org/10.1111/ijfs.13164

GYLES CL. 2007. Shiga toxin-producing Escherichia coli: an overview. Journal of Animal Science. 85: 45-62. https://doi.org/10.2527/jas.2006-508

IWERIEBOR BC, Iwu CJ, Obi LC, Nwodo UU, Okoh Al 2015. Multiple antibiotic resistances among Shiga toxin producing Escherichia coli 0157 in feces of dairy cattle farms in Eastern Cape of South Africa. BMC microbiology. 15(1):213. https://bmcmicrobiol.biomedcentral.com/articles/10.1186/s12866-015-0553-y

HIRVONEN JJ, Siitonen A, Kaukoranta SS. 2012. Usability and performance of CHROMagar STEC in detection of Shiga toxin-producing Escherichia coli strains. J Clin Microbiol. 50:3586-3590. https://doi.org/10.1128/JCM.01754-12

KAPER JB, Nataro JP, Mobley HLT. 2004. Pathogenic Escherichia coli. Nat Rev Microbiol. 2:123-40. https://www.nature.com/articles/nrmicro818

KIM YB, Seo KW, Shim JB, Son SH, Noh EB, Lee Y J. 2019. Molecular characterization of antimicrobial-resistant Enterococcus faecalis and Enterococcus faecium isolated from layer parent stock. Poultry science. 98(11):5892-5899. https://doi.org/10.3382/ps/pez288

LARA DJA, Silva VM, Bañuelos VR, Delgadillo RL, Delgadillo RO. 2019. Incidencia de Escherichia coli O157: $\mathrm{H} 7$ en heces de rumiantes lactantes con síndrome diarreico. Revista MVZ Córdoba. 24(3): 7339-7345. https://doi.org/10.21897/rmvz.1232 
LÓPEZ MA, Valbuena-Gregorio E, Quihui-Cota L, Morales-Figueroa GG, Ruiz-Cruz S, Campos-García JC, Díaz-Meza E, Pablos-Rodríguez DE. 2017. Efecto de Microemulsiones de Aceites Esenciales Sobre el Eritrocito Humano y Bacterias Patógenas. Revista mexicana de ingeniería biomédica. 38(1):247-254 . http://www.rmib.mx/index.php/rmib/article/view/27

LOZOYA X, Reyes MH, Chávez MA., Martínez GMC, Soto GY and Doubova SV. 2002. Intestinalanti-spasmodic effect of a phytodrug of Psidium guajava folia in the treatment ofacute diarrheic disease. Journal of Ethnopharmacology. 83(1-2): 19-24. https://doi.org/10.1016/S0378-8741(02)00185-X

MARTÍNEZ MJ, Molina N, Boucourt E. 1997. Evaluación de la actividad antimicrobiana de Psidium guajava L. (guayaba). Revista Cubana de Plantas Medicinales. 2(1): 12-14. http://scielo.sld.cu/scielo.php?pid=S1028-

47961997000100003\&script=sci_arttext\&tIng=en

MEIRA NV, Holley RA, Bordin K, de Macedo RE, Luciano FB. 2017. Combination of essential oil compounds and phenolic acids against Escherichia coli O157: $\mathrm{H} 7$ in vitro and in dry-fermented sausage production. International journal of food microbiology. 260:5964. https://doi.org/10.1016/j.ijfoodmicro.2017.08.010

MILTON AAP, Agarwal RK, Priya GB, Aravind M, Athira CK, Rose L, Saminathan M, Sharma AK, Kumar A. 2018. Captive wildlife from India as carriers of Shiga toxinproducing, Enteropathogenic and Enterotoxigenic Escherichia coli. Journal of Veterinary Medical Science. 81(2): 321-327. https://doi.org/10.1292/jvms.18-0488

MOYNE AL, Sudarshana MR, Blessington T, Koike ST, Cahn MD, Harris LJ. 2011. Fate of Escherichia coli O157: H7 in field-inoculated lettuce. Food Microbiology. 28(8): 14171425. https://doi.org/10.1016/j.fm.2011.02.001

NILE SH, Nile AS, Keum YS. 2017. Total phenolics, antioxidant, antitumor, and enzyme inhibitory activity of Indian medicinal and aromatic plants extracted with different extraction methods. 3 Biotech. 7(1): 1-10. https://ink.springer.com/article/10.1007/s13205-0170706-9

PARSONS BD, Zelyas N, Berenger BM, Chui L. 2016. Detection, characterization, and typing of Shiga toxin-producing Escherichia coli. Frontiers in microbiology. 7: 1-12. https://doi.org/10.3389/fmicb.2016.00478 
PESEWU GA, Cutler RR, Humber DP. 2008. Antibacterial activity of plants used in traditional medicines of Ghana with particular reference to MRSA. Journal of ethnopharmacology. 116(1): 102-111. https://doi.org/10.1016/j.jep.2007.11.005

RATTANACHAIKUNSOPON P, Phumkhachorn P. 2010. Contents and antibacterial activity of flavonoids extracted from leaves of Psidium guajava. Journal of Medicinal Plants Research. 4(5): 393-396. https://doi.org/10.5897/JMPR09.485

RODRÍGUEZ-ANGELES G. 2002. Principales características y diagnóstico de los grupos patógenos de Escherichia coli. Salud pública de México. 44: 464-475. http://www.insp.mx/salud/index.html

RODRÍGUEZ RA, Lafourcade PA, Pérez RL. 2013. Hojas de Psidium guajava L. Revista Cubana de Farmacia. 47(1): 127-135.

https://www.researchgate.net/profile/Jesus_Rafael_Rodriguez_Amado/publication/2607 74883_Hojas_de_Psidium_guajava_L/links/55fae20008ae07629e07b496/Hojas-dePsidium-guajava-L.pdf

TANG Y, Kim H, Singh AK, Aroonnual A, Bae E, Rajwa B, Bhunia AK. 2014. Light scattering sensor for direct identification of colonies of Escherichia coli serogroups O26, O45, O103, O111, 0121, O145 and 0157. PLoS One. 9(8): e105272. https://doi.org/10.1371/journal.pone.0105272

VITAS AI, Naik D, Pérez-Etayo L, González D. 2018. Increased exposure to extendedspectrum $\beta$-lactamase-producing multidrug-resistant Enterobacteriaceae through the consumption of chicken and sushi products. International journal of food microbiology. 269: 80-86. https://doi.org/10.1016/j.ijfoodmicro.2018.01.026

WANG Y, Yam KL. 2018. Inhibitory effect of thymol via different modes of delivery on growth of Escherichia coli DH5a. Food Packaging and Shelf Life. 16:92-96. https://doi.org/10.1016/j.fpsl.2018.02.007

WITTUM TE, Mollenkopf DF, Daniels JB, Parkinson AE, Mathews JL, Fry PR, Abley MJ, Gebreyes WA. 2010. CTX-M-type expendedspectrum b-lactamases present in Escherichia coli from the feces of cattle in Ohio, United States. Foodborne Pathog Dis. 7:1575-9. https://doi.org/10.1089/fpd.2010.0615 